\title{
Retropupillary iris claw intraocular lens implantation in aphakia for dislocated intraocular lens [Corrigendum]
}

Faria MY, Ferreira NP, Pinto JM. Int Med Case Rep J. 2016 Aug 29;9:261-5.

On page 261, Ivo Gama should have been listed as an author.

The incorrect author list was:

$$
\begin{aligned}
& \text { Mun Yueh Faria } \\
& \text { Nuno Pinto Ferreira } \\
& \text { Joana Medeiros Pinto } \\
& \text { David Cordeiro Sousa } \\
& \text { Ines Leal } \\
& \text { Eliana Neto } \\
& \text { Carlos Marques-Neves }
\end{aligned}
$$

The correct author list should have been:

$$
\begin{aligned}
& \text { Mun Yueh Faria }{ }^{1-3} \\
& \text { Nuno Pinto Ferreira }{ }^{1-3} \\
& \text { Joana Medeiros Pinto } \\
& \text { Ivo Gama } \\
& \text { David Cordeiro Sousa } \\
& \text { Ines Leal'-3 } \\
& \text { Eliana Neto } \\
& \text { Carlos Marques-Neves }
\end{aligned}
$$

\section{Publish your work in this journal}

The International Medical Case Reports Journal is an international, peer-reviewed open-access journal publishing original case reports from all medical specialties. Previously unpublished medical posters are also accepted relating to any area of clinical or preclinical science. Submissions should not normally exceed 2,000 words or
4 published pages including figures, diagrams and references. The manuscript management system is completely online and includes a very quick and fair peer-review system, which is all easy to use. Visit $\mathrm{http}: / / \mathrm{www}$. dovepress.com/testimonials.php to read real quotes from published authors.

Submit your manuscript here: https://www.dovepress.com/international-medical-case-reports-journal-journal 